\title{
Gusanos perforadores (Sipuncula y Annelida: Polychaeta) de los arrecifes coralinos del Pacífico Tropical Oriental (isla Gorgona, Colombia)
}

\author{
Boring worms (Sipuncula and Annelida: Polychaeta) from Tropical \\ Eastern Pacific coral reefs (Gorgona Island, Colombia)
}

\author{
María Fernanda Cardona-Gutiérrez ${ }^{1,2}$ y Edgardo Londoño-Cruz ${ }^{l}$ * \\ (iD) $0000-0003-4910-0312$ \\ (D) $0000-0001-5762-9430$ \\ 1. Grupo de Investigación en Ecosistemas Rocosos Intermareales y Submareales Someros (LITHOS), Departamento de Biología, Universidad del Valle, \\ Cali,Colombia.maria.cardona.gutierrez@correounivalle.edu.co; edgardo.londono@correounivalle.edu.co* \\ 2. Centro Interdisciplinario de Ciencias Marinas (Cicimar), Instituto Politécnico Nacional, La Paz, Baja California Sur, México. \\ * Autor de correspondencia.
}

\section{RESUMEN}

$\mathrm{E}$ conocimiento de los gusanos bioerosionadores en los arrecifes coralinos del Pacífico Tropical Oriental (PTO) colombiano es escaso. Para saber qué especies perforan estos sustratos coralinos se utilizaron unidades experimentales hechas de ramas muertas de Pocillopora spp., las cuales estuvieron expuestas en los arrecifes coralinos de la isla Gorgona (Pacífico colombiano) durante varios periodos de tiempo. Un total de 571 gusanos de 21 especies fueron recolectados; de estas, 18 son poliquetos y 3 sipúnculos. La mayoría de estas especies corresponden a nuevos registros para el PTO colombiano, en concreto, 13 de las 18 especies de poliquetos y 2 de las 3 de sipúnculos. Estos hallazgos sugieren la necesidad de realizar más estudios con el objetivo de generar conocimiento sobre la biodiversidad de estos grupos poco conocidos en regiones poco estudiadas, como es el caso del PTO colombiano.

PALABRAS CLAVE: Pacífico colombiano, perforadores, carbonato de calcio, nuevos registros, gusanos

\section{ABSTRACT}

$\mathrm{T}$

The knowledge of boring worms in the coral reefs of the Colombian Tropical Eastern Pacific (TEP) is scarce. To know which species bore into coralline substrates, experimental units made of dead branches of Pocillopora spp. were deployed and exposed in the coral reefs of Gorgona Island (Colombian Pacific) during various periods of time. A total of 571 worms in 21 species were collected. Of these, 18 were polychaetes and 3 sipunculids. Most of these species correspond to new records in the Colombian TEP, specifically 13 of the 18 species of polychaetes and 2 of the 3 species of sipunculids. These findings suggest that more research is needed to gain knowledge about the biodiversity of these poorly-known groups in understudied regions like the Colombian TEP.

KEYWORDS: Colombian Pacific, borers, calcium carbonate, new records, worms

DOI: https://doi.org/10.25268/bimc.invemar.2020.49.2.924 


\section{INTRODUCCIÓN}

Los gusanos (poliquetos y sipúnculos) asociados a los arrecifes coralinos del Parque Nacional Natural Gorgona (PNN Gorgona) (Figura 1) son poco conocidos, principalmente por la dificultad de observarlos debido al hábitat que ocupan o por la dificultad de recolectarlos. Los estudios en los que se han mencionado algunos de estos organismos han sido pocos (v. g. Cantera et al., 2003; Londoño-Cruz et al., 2003) y, por lo tanto, son escasas las especies que se han identificado. Algunos gusanos son considerados perforadores (Hutchings, 1986), i. e. penetran (usando diferentes medios) el sustrato (v. g. coral) que les sirve como refugio seguro. Las galerías que producen pueden debilitar la resistencia del sustrato, lo cual lo hace más frágil y susceptible de romperse; cuando estas galerías quedan vacías, pueden servir de entrada a otras especies perforadoras (v. g. moluscos bivalvos) (LondoñoCruz et al., 2003), lo que incrementa la destrucción coralina.

El PNN Gorgona $\left(2,9689^{\circ} \mathrm{N}-78,1819^{\circ} \mathrm{W}\right)$, junto con el islote Gorgonilla, es el territorio insular más extenso sobre la plataforma continental del Pacífico colombiano. Esta isla está ubicada a $\sim 30 \mathrm{~km}$ de la costa (Zapata, 2001; Londoño-Cruz, et al., 2003; Giraldo, 2012). Las condiciones para la formación de arrecifes coralinos son marginales. Las precipitaciones anuales son altas (promedio de $8000 \mathrm{~mm} /$ año), lo que ocasiona bajas salinidades que fluctúan entre 29 (marzo) y 31 (septiembre). La temperatura media superficial del agua presenta variación estacional y es influenciada por fenómenos naturales como El Niño Oscilación Sur $\left(18{ }^{\circ} \mathrm{C}\right.$ en meses de La Niña y $33{ }^{\circ} \mathrm{C}$ en meses de El Niño). Las mareas son semidiurnas, con amplio rango mareal $(\sim 4,5 \mathrm{~m})$. Finalmente, se presenta una reducción en la transparencia del agua (poca visibilidad), dado que la concentración de sólidos disueltos totales es relativamente alta: esta varía $\sim 8 \mathrm{~m}$ a lo largo del año, con valores extremadamente bajos durante lluvias torrenciales (Prahl y Erhardt, 1985; Zapata, 2001; Londoño-Cruz et al., 2003; Giraldo et al., 2008). A pesar de esto, el PNN Gorgona presenta algunos de los arrecifes coralinos más desarrollados y diversos del límite sur de la distribución de arrecifes coralinos del PTO (Zapata, 2001; Londoño-Cruz et al., 2003; Zapata y Vargas-Ángel, 2003; Giraldo et al., 2008).

Los principales y más grandes arrecifes de coral del PNN Gorgona, La Azufrada y Playa Blanca (9,8 y 9,4 ha, respectivamente), están ubicados al costado oriental de la isla. Estos arrecifes son jóvenes y someros (Zapata, 2001; Giraldo et al., 2008) y están dominados por especies de corales

\section{INTRODUCTION}

The worms (polychaetes and sipunculids) inhabiting the coral reefs of the Gorgona National Natural Park (Gorgona) (Figure 1) in Colombia are poorly known, mainly due to the difficulty of direct observation given the habitats they use or to the difficulty of collecting these animals. There are few studies (e.g. Cantera et al., 2003; Londoño-Cruz et al., 2003) that have mentioned some of these organisms, hence, only a few species have been identified. Some worms are considered borers (Hutchings, 1986), i.e. they penetrate (by different means) the substrate (e.g. coral) to dwell in a safe refugium. The galleries produced may weaken the substrate hardness and hence make it more fragile and prone to break; when empty these galleries may also serve as openings for the colonization of other boring species (e.g. bivalve mollusks) (Londoño-Cruz et al., 2003), which increases coral destruction.

Gorgona $\left(2.9689{ }^{\circ} \mathrm{N}-78.1819{ }^{\circ} \mathrm{W}\right)$, along with Gorgonilla islet, is the largest insular territory on the Colombian Pacific coast. This island is $\sim 30 \mathrm{~km}$ from the closest mainland point (Zapata, 2001; Londoño-Cruz, et al., 2003; Giraldo, 2012). Environmental conditions are marginal to coral reef formations. The mean annual precipitation is $8000 \mathrm{~mm}$ with concomitant low salinities that vary between 29 (during March) and 31 (during September). Water temperature varies annually and is affected by the ENSO cycle, reaching highs of $33^{\circ} \mathrm{C}$ during El Niño and lows of $18{ }^{\circ} \mathrm{C}$ during La Niña. Tides are semidiurnal with a range of $\sim 4.5 \mathrm{~m}$. Finally, the concentration of total dissolved solids is relatively high, which is reflected in reduced water transparency (low visibility), this varies around $8 \mathrm{~m}$ yearround, with extremely low values during periods of heavy rain (Prahl and Erhardt, 1985; Zapata, 2001; LondoñoCruz et al., 2003; Giraldo et al., 2008). Regardless of these conditions, Gorgona holds some of the most developed and diverse coral reefs in the southern reach of coral reef distribution in the TEP (Zapata, 2001; Londoño-Cruz, et al., 2003; Zapata and Vargas-Ángel, 2003; Giraldo et al., 2008).

The largest coral reefs of Gorgona, Playa Blanca and La Azufrada (9.8 and 9.4 ha, respectively), are located on the lee (southeastern side) of the island. These reefs are young and shallow (Zapata, 2001; Giraldo et al., 2008), and are dominated by Indopacific coral species (Zapata and Morales, 1997; Zapata, 2001). Playa Blanca is formed by two reef patches, the smallest of which is located to the north; in turn, La Azufrada is north to Playa Blanca (Prahl 
provenientes del Indopacífico (Zapata y Morales, 1997; Zapata, 2001). Playa Blanca está conformado por dos parches, el más pequeño de los cuales está hacia el norte. Por su parte, La Azufrada está al norte de Playa Blanca (Prahl y Erhardt, 1985; Zapata, 2001) (Figura 1). Estos arrecifes muestran el típico patrón de zonación de los arrecifes coralinos de borde, con un canal de navegación, seguido del trasarrecife, donde la cobertura coralina es menos de $50 \%$; la planicie arrecifal, el frente arrecifal, donde la cobertura coralina es la mayor (alcanza más de $80 \%$ ), y el talud arrecifal, donde la cobertura coralina baja hasta $20 \%$, con abundante cascajo coralino (Prahl y Erhardt, 1985; Díaz et al., 2000; Zapata, 2001). La riqueza de corales en el PNN Gorgona es la más alta en el Pacífico colombiano, con dominancia de corales del género Pocillopora (Prahl y Erhardt, 1985; Zapata, 2001). Dada la importancia de los ecosistemas de arrecifes de coral, la escasez en el conocimiento de los gusanos de la costa del Pacífico de and Erhardt, 1985; Zapata, 2001) (Figure 1). These reefs show the typical zonation pattern of fringing coral reefs, with a navigation channel, followed by the back-reef, where live coral cover is less than $50 \%$; the reef-flat, the reef-front, where live coral cover is highest, reaching more than $80 \%$, and the reef-slope, where live coral cover is as low as $20 \%$ and there is abundant coral rubble (Prahl and Erhardt, 1985; Díaz et al., 2000; Zapata, 2001). Although coral richness in Gorgona is the highest in the Colombian Pacific, there is a dominance of corals of the genus Pocillopora (Prahl and Erhardt, 1985; Zapata, 2001). Given the importance of coral reef ecosystems, the scarcity in knowledge regarding worms in the Pacific coast of Colombia, and that certain species of worms may act as borers of coral substrates, the main purpose of this document was to identify the members of the boring worms associated to coral reefs of Gorgona Island (Colombian Pacific).

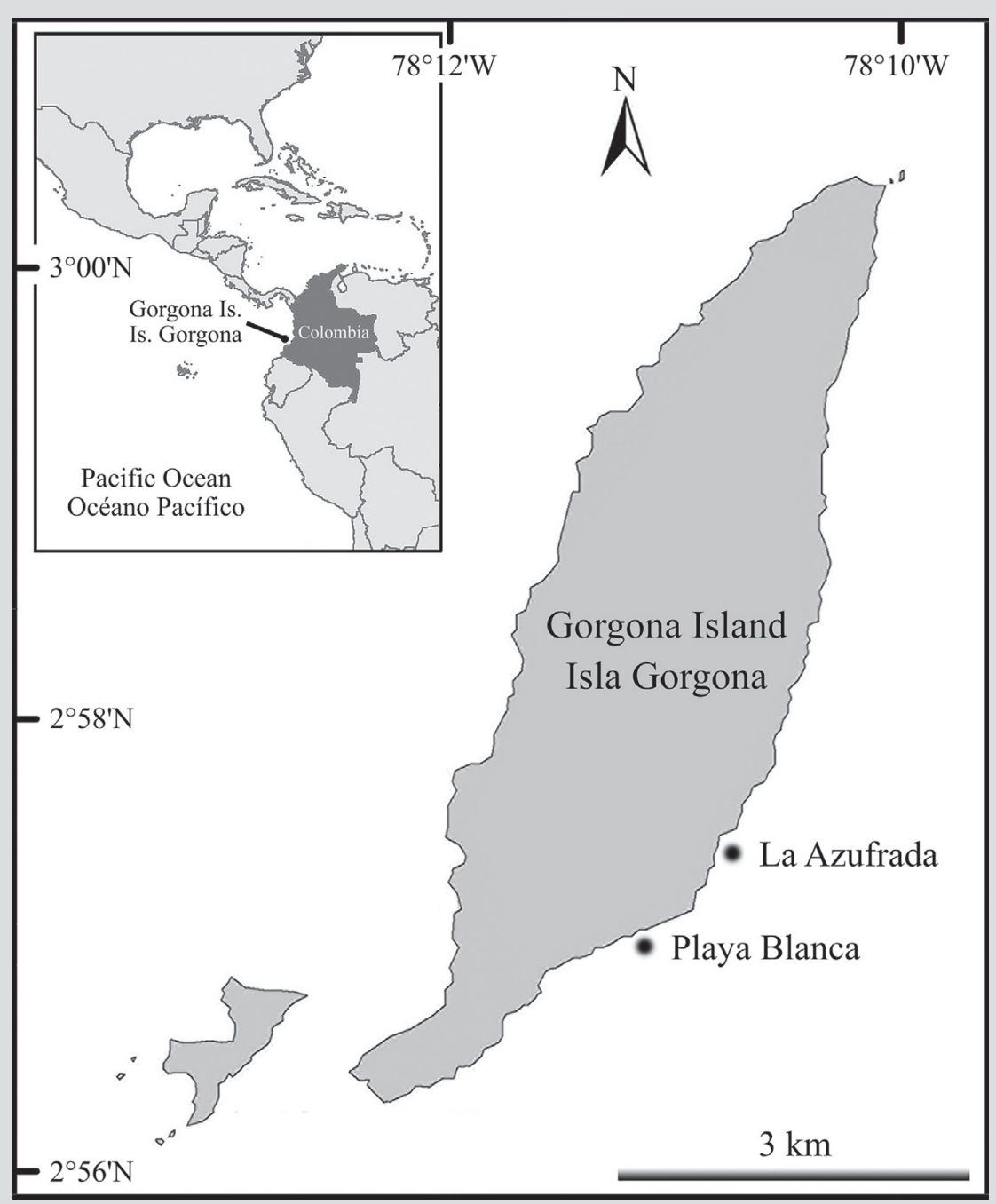

Figura 1. Ubicación de la isla Gorgona en el POT y de los dos arrecifes coralinos: Playa Blanca y La Azufrada, donde se recolectaron los gusanos.
Figure 1. Location of Gorgona Island in the TEP and the two coral reefs: Playa Blanca and La Azufrada, from which worms were collected. 
Colombia y que ciertas especies de gusanos pueden actuar como perforadores de sustratos de coral, el objetivo principal de este documento fue identificar a los gusanos perforadores asociados a los arrecifes de coral de la isla Gorgona (Pacífico colombiano).

\section{MATERIALES Y MÉTODOS}

Se recolectaron ramas muertas, sin señales de bioerosión, de Pocillopora spp. (aquellas que presentaron señales de bioerosión fueron descartadas) y se les dio forma de cilindros, con una pulidora manual, de tamaño similar (Unidades Experimentales, UE). A cada UE se le perforó un extremo, en el que se introdujo y cementó (con resina epóxica) una varilla de acero inoxidable de $1,59 \mathrm{~mm}$ de diámetro en forma de L. Además, varillas de acero inoxidable más grandes $(6,35 \mathrm{~mm}$ de diámetro $\times 2 \mathrm{~m}$ de largo $)$ fueron clavadas en la matriz arrecifal, lo que dejó $50 \mathrm{~cm}$ por fuera de esta. Las UE se ataron, utilizando amarraderas plásticas, a esta porción de la varilla (una UE por varilla). Cinco UE fueron puestas de forma aleatoria en cada zona de cada arrecife y se dejaron allí durante uno de cinco diferentes periodos de exposición que fluctuaron entre 6 y 18 meses (con intervalos de 3 meses). De esta manera, se logró una buena representatividad temporal y espacial. Todas las UE $(n=200)$ fueron puestas el mismo día (2016/02/11). Cuando el periodo de exposición para una UE terminó, esta se recolectó, se almacenó en un recipiente plástico con formalina al $10 \%$ y se llevó al laboratorio. Todos los organismos adheridos a la superficie de las UE fueron removidos. Después de esto, cada UE fue cuidadosamente fracturada usando alicates de presión con el propósito de extraer todos los organismos presentes en el interior del fragmento de coral. Todos los gusanos (poliquetos y sipúnculos) fueron identificados al nivel taxonómico más bajo posible usando claves dicotómicas (Cutler, 1994; León-González et al., 2009); además, se revisaron las descripciones originales disponibles en Biodiversity Heritage Library. Finalmente, todas las especies identificadas fueron almacenadas en la Colección de Referencia de Organismos Marinos (CROM) del Departamento de Biología de la Universidad del Valle, Cali (Colombia).

\section{RESULTADOS}

Se colectaron en total 571 individuos de gusanos (poliquetos + sipúnculos) (Tabla 1). Estos animales pertenecen a 21 especies, 18 de poliquetos y 3 de sipúnculos (ver Figura 2 para un ejemplo de algunas de las especies colectadas). Las

\section{MATERIALS AND METHODS}

Branches of Pocillopora spp. colonies, with no signs of bioerosion, were collected and shaped into similar-sized cylinders (Experimental Units: EUs), using a handheld grinder. Each EU was drilled in one of the ends to anchor a $1.59 \mathrm{~mm}$ diameter stainless steel L-shaped rod using epoxy resin. Besides, larger $(6.35 \mathrm{~mm}$ diameter $\times 2 \mathrm{~m}$ long) stainless steel rods were hammered, leaving around $50 \mathrm{~cm}$ outside, into the reef framework. The EUs were tied (using cable ties) to this portion of the rod (one EU per rod). Five EUs were randomly deployed along each reef zone of each reef and left there during one of five different exposure periods that ranged between 6 and 18 months (with 3 month intervals). In this manner, a good spatial and temporal representation was achieved. All EUs $(n=200)$ were deployed the same day $(2016 / 02 / 11)$. Once the exposure period ended for an EU, it was collected, stored in a plastic container with $10 \%$ formalin, and taken to the lab. All fouling was removed from the EU surface. After that, each EU was carefully fragmented using locking pliers to extract all organisms dwelling inside the coral matrix. All worms (polychaetes and sipunculids) were identified to the lowest taxonomic level possible using dichotomic keys (Cutler, 1994; de León-González et al., 2009). Besides, original descriptions available in the Biodiversity Heritage Library were reviewed. Finally, all identified species were stored in the Reference Collection of Marine Organisms (CROM) of the Department of Biology at the Universidad del Valle, Cali (Colombia).

\section{RESULTS}

A total of 571 worm specimens (polychaetes + sipunculids) were collected (Table 1). These worms were allocated in 21 species: 18 polychaetes and 3 sipunculids (see Figure 2 for an example of some of the collected species). Polychaetes comprise 10 families and sipunculids comprise 2. New records are marked with superscripts as follows: ${ }^{1}$ for Gorgona coral reefs only, ${ }^{2}$ for PNN Gorgona, and ${ }^{3}$ for the Colombian Eastern Tropical Pacific. The consulted literature for each species is listed right below the species name. The material examined presents the batch code of the CROM. All information related to distribution was obtained from the World Register of Marine Species (WoRMS, 2019). Finally, a brief discussion is added only to species that match original descriptions but differ in certain characters. 
especies de poliquetos se enmarcaron en 10 familias y las de sipúnculos en 2 familias.

Los nuevos registros son marcados con superíndices: ${ }^{1}$ cuando el registro es solo para los arrecifes del PNN Gorgona, ${ }^{2}$ para el PNN Gorgona y ${ }^{3}$ para el Pacífico Oriental Tropical colombiano. El material bibliográfico consultado para cada especie se lista justo debajo del nombre de la especie. El material examinado presenta el código de ingreso al CROM. La información de la distribución de cada especie fue consultada en la plataforma del World Register or Marine Species (WoRMS, 2019). Finalmente, se realizó una breve discusión solo para aquellas especies que cumplieron con la descripción original, pero difirieron en unos pocos caracteres.

Tabla 1. Número total de especímenes de cada especie en cada arrecife coralino y zona arrecifal (BR: Trasarrecife, RP: Planicie arrecifal; RF: Frente arrecifal y RS: Talud arrecifal) recolectados en la isla Gorgona (POT colombiano) durante cinco períodos de exposición diferentes.
Table 1. Total number of specimens of each species from each coral reef and reef zone (BR: Back-reef, RP: Reef-flat, RF: Reef-front, and RS: Reef-slope) collected in Gorgona Island (Colombian ETP) during the five different exposure periods.

\begin{tabular}{|c|c|c|c|c|c|c|c|c|c|}
\hline \multicolumn{2}{|r|}{ Taxonomy/Taxonomía } & \multicolumn{4}{|c|}{ La Azufrada } & \multicolumn{4}{|c|}{ Playa Blanca } \\
\hline Family/Familia & Species/Especies & BR & $\mathbf{R P}$ & $\mathbf{R F}$ & RS & BR & $\mathbf{R P}$ & RF & RS \\
\hline Amphinomidae & Chloeia entypa & & & & & & 1 & & \\
\hline \multirow{3}{*}{ Cirratulidae } & Dodecaceria fewkesi & 3 & & & 2 & 5 & & & 1 \\
\hline & Dodecaceria laddi & 4 & & & 11 & 3 & 2 & 1 & 3 \\
\hline & Timarete luxuriosa & & 1 & 1 & & & & & \\
\hline Dorvilleidae & Dorvillea rubra & 17 & 8 & 8 & 1 & 21 & 15 & 9 & 4 \\
\hline \multirow{3}{*}{ Eunicidae } & Eunice cedroensis & 1 & & 3 & & & 2 & 1 & 1 \\
\hline & Lysidice ninetta & 1 & & & & & & 1 & 3 \\
\hline & Palola paloloides & & & & 1 & & & & \\
\hline Flabelligeridae & Semiodera cariboum & & & & 3 & & & & \\
\hline Phyllodocidae & Eteone cf. pacifica & & 1 & & 1 & & & & \\
\hline Sabellidae & Euratella salmacidis & 5 & 4 & 1 & 4 & 2 & 2 & 9 & 11 \\
\hline \multirow{2}{*}{ Spionidae } & Dipolydora commensalis & 43 & 7 & 5 & 4 & 17 & 9 & 3 & 9 \\
\hline & Polydora ciliata & 1 & & & & & & & \\
\hline \multirow{4}{*}{ Syllidae } & Cicese cf. sphaerosylliformis & & & & & & & & 1 \\
\hline & Dentatisyllis cf. carolinae & & & 2 & & & & & 1 \\
\hline & Syllis gracilis & 15 & 29 & 24 & 14 & 7 & 16 & 18 & 4 \\
\hline & Syllis prolifera & & & & & & 1 & & \\
\hline Aspidosiphonidae & Aspidosiphon (Aspidosiphon) cf. elegans & 10 & 9 & & 5 & 13 & 21 & 4 & 1 \\
\hline \multirow{2}{*}{ Phascolosomatidae } & Phascolosoma (Phascolosoma) cf. nigrescens & & & 1 & & & 1 & 1 & \\
\hline & Phascolosoma (Phascolosoma) cf. perlucens & 17 & 19 & 8 & 12 & 9 & 10 & 14 & 8 \\
\hline \multicolumn{2}{|c|}{ Total per zone and reef/Total por zona y arrecife } & 117 & 78 & 53 & 58 & 77 & 80 & 61 & 47 \\
\hline \multicolumn{2}{|c|}{ Total per reef/Total por arrecife } & \multicolumn{4}{|c|}{306} & \multicolumn{4}{|c|}{265} \\
\hline \multicolumn{2}{|l|}{ Grand total/Gran total } & \multicolumn{8}{|c|}{571} \\
\hline
\end{tabular}




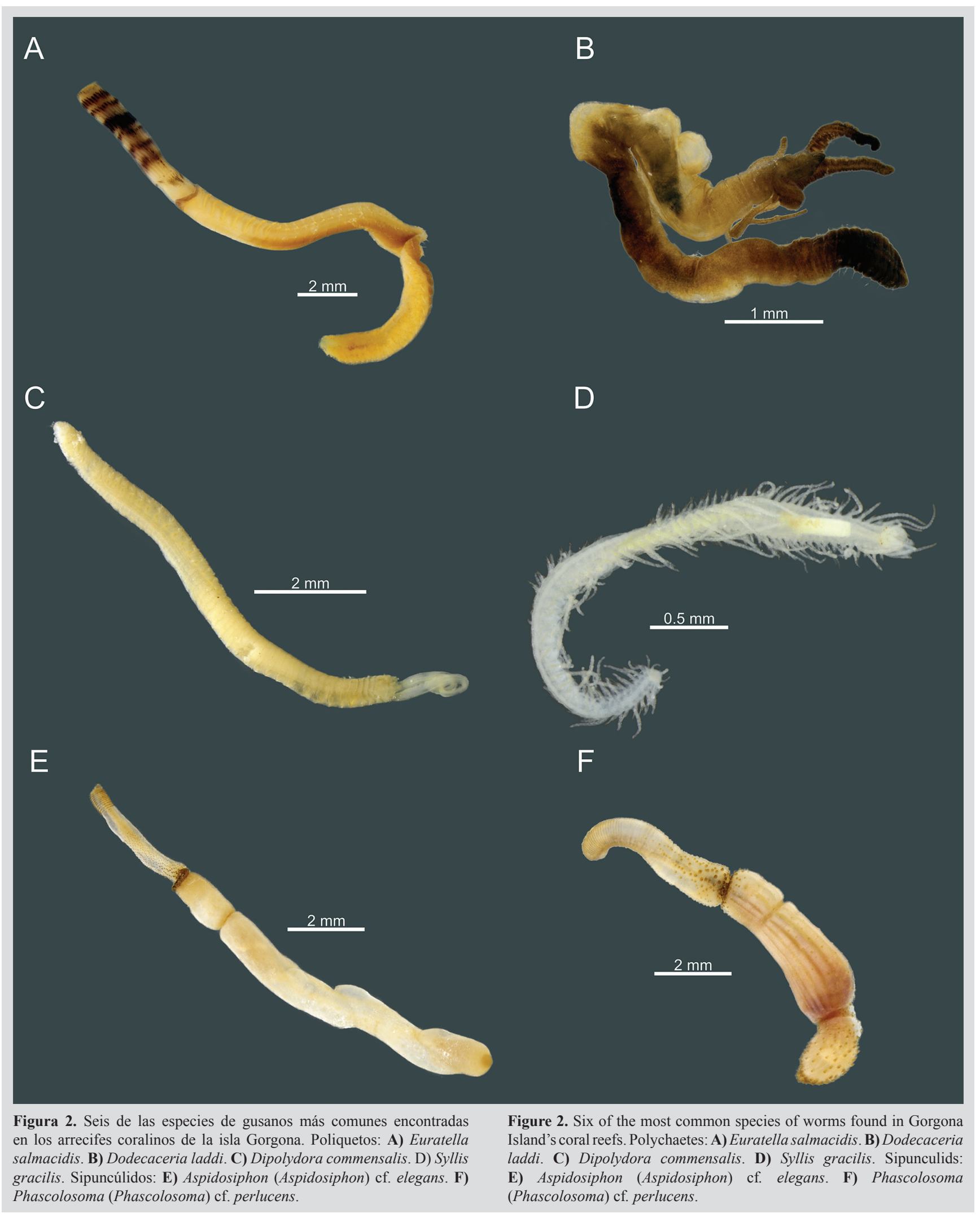




\section{Annelida}

\section{Clase Polychaeta}

Familia Amphinomidae Lamarck, 1818

Género Chloeia Lamarck, 1818

Chloeia entypa ${ }^{1}$ Chamberlin, 1919

Chloeia entypa: Chamberlin, 1919; Kudenov, 1975;

Fauchald, 1977; Prahl et al., 1979; Laverde-Castillo, 1986; Hernández-Alcántara y Solís-Weiss, 1991; Salazar-Vallejo y Londoño-Mesa, 2004; Valencia et al., 2014.

Material examinado: APN0001.

Distribución: Pacífico (México) y PTO.

Discusión: El espécimen coincide con la descripción de Kudenov (1975), quien menciona la presencia de neurosetas bifurcadas y serradas en la región posterior del cuerpo. Sin embargo, de León-González et al. (2009) dicen que las antenas laterales están pigmentadas. Tal pigmentación no se observó, posiblemente debido a que el espécimen la perdió por el método de fijación. Se cree que el individuo observado puede ser un juvenil por la cantidad de segmentos que presenta (14).

Familia Cirratulidae Ryckholt, 1851

Género Dodecaceria Örsted, 1843

Dodecaceria fewkesi $i^{2}$ Berkeley \& Berkeley, 1954

Dodecaceria fewkesi: Berkeley y Berkeley, 1954; SalazarVallejo y Londoño-Mesa, 2004.

Material examinado: CIR0026, CIR0027, CIR0028, CIR0029.

Distribución: Sur de California, bahía Coos.

Género Dodecaceria Örsted, 1843

Dodecaceria laddi ${ }^{3}$ Hartman, 1954

Dodecaceria laddi: Hartman, 1954; Gibson, 1978; White, 1980; Abd-Elnaby, 2009; Magalhaes y Bailey-Brock, 2013.

Material examinado: CIR0030, CIR0031, CIR0032, CIR0033, CIR0034, CIR0035.

Distribución: bahía de Kaneohe (Hawái), islas Marshall, Pulo Boenda (Sumatra).

Discusión: El espécimen coincide con la descripción de Hartman (1954); sin embargo, en las setas de los primeros siete segmentos no fue posible observar el limbo (Hartman, 1954, Figura 177H). Esto pudo deberse a la posición de las setas.

\section{Annelida}

\section{Class Polychaeta}

Family Amphinomidae Lamarck, 1818

Genus Chloeia Lamarck, 1818

Chloeia entypa ${ }^{1}$ Chamberlin, 1919

Chloeia entypa: Chamberlin, 1919; Kudenov, 1975;

Fauchald, 1977; Prahl et al., 1979; Laverde-Castillo, 1986; Hernández-Alcántara y Solís-Weiss, 1991; Salazar-Vallejo y Londoño-Mesa, 2004; Valencia et al., 2014.

Material examined: APN0001.

Distribution: Pacific (Mexico) and TEP.

Discussion: The specimen matched the description by Kudenov (1975), who mentions the presence of serrate and bifurcate neurosetae in the posterior part of the body. Although, de León-González et al. (2009) mention that lateral antennae are pigmented. This pigmentation was not observed, probably because it was lost due to the fixation method used. It is highly probable that the specimen is a juvenile due to the number of segments present (14).

Family Cirratulidae Ryckholt, 1851

Genus Dodecaceria Örsted, 1843

Dodecaceria fewkesi ${ }^{2}$ Berkeley \& Berkeley, 1954

Dodecaceria fewkesi: Berkeley y Berkeley, 1954; SalazarVallejo y Londoño-Mesa, 2004.

Material examined: CIR0026, CIR0027, CIR0028, CIR0029.

Distribution: South of California, Coos Bay.

Genus Dodecaceria Örsted, 1843

Dodecaceria laddi ${ }^{3}$ Hartman, 1954

Dodecaceria laddi: Hartman, 1954; Gibson, 1978; White, 1980; Abd-Elnaby, 2009; Magalhaes y Bailey-Brock, 2013.

Material examined: CIR0030, CIR0031, CIR0032, CIR0033, CIR0034, CIR0035.

Distribution: Kaneohe Bay (Hawaii), Marshall Islands, Pulo Boenda (Sumatra).

Discussion: The specimens matched the description by Hartman (1954); however, it was not possible to observe the limbo that appears in the setae of the first seven segments (Hartman, 1954, Figure 177H). This might be due to the position of the setae. 
Género Timarete Kinberg, 1866

Timarete luxuriosa ${ }^{3}$ (Moore, 1904)

Timarete luxuriosa: Moore, 1904; Hernández-Alcántara y Solís-Weiss, 1991; Hernández-Alcántara, 2003; SalazarVallejo y Londoño-Mesa, 2004; Choi et al., 2018.

Material examinado: CIR0036, CIR0037.

Distribución: golfo de California, Pacífico mexicano, PTO.

Familia Dorvilleidae Chamberlin, 1919

Género Dorvillea Parfitt, 1866

Dorvillea rubra ${ }^{3}$ (Grube, 1856)

Dorvillea rubra: Grube, 1856; Perkins, 1998; Salazar-

Vallejo y Londoño-Mesa, 2004; Hernández-Alcántara et al., 2014.

Material examinado: DOR0001, DOR0002, DOR0003, DOR0003, DOR0004, DOR0005, DOR0006.

Distribución: Caribe, Florida, golfo de México, PTO.

Familia Eunicidae Berthold, 1827

Género Eunice Cuvier, 1817

Eunice cedroensis ${ }^{3}$ Fauchald, 1970

Eunice cedroensis: Fauchald, 1970; Salazar-Vallejo y

Londoño-Mesa, 2004.

Material examinado: EUN0035, EUN0036, EUN0037, EUN0038.

Distribución: golfo de México, PTO.

Discusión: El espécimen coincide con la descripción de Fauchald (1970); sin embargo, no se observaron las setas ilustradas por el autor en la Figura E (Plancha 2).

Género Lysidice Lamarck, 1818

Lysidice ninetta ${ }^{1}$ Audouin \& H Milne Edwards, 1833

Lysidice ninetta: Audouin y Milne, 1833; Monro, 1928;

Fauchald, 1970, 1977; Laverde-Castillo, 1986; Hernández-

Alcántara y Solís-Weiss, 1991; de León-González et al., 1993; Gómez et al., 1997; Cantera et al., 1999; Salazar-

Vallejo y Londoño-Mesa, 2004.

Material examinado: EUN0040, EUN0041, EUN0042

Distribución: PTO, Colombia, golfo de México, Cuba,

Belice, Bélgica, mar Caribe, Francia, Grecia, Jamaica,

Nueva Zelanda, mar Rojo, España, Reino Unido.
Genus Timarete Kinberg, 1866

Timarete luxuriosa ${ }^{3}$ (Moore, 1904)

Timarete luxuriosa: Moore, 1904; Hernández-Alcántara y Solís-Weiss, 1991; Hernández-Alcántara, 2003; SalazarVallejo y Londoño-Mesa, 2004; Choi et al., 2018.

Material examined: CIR0036, CIR0037.

Distribution: Gulf of California, Mexican Pacific, TEP.

Family Dorvilleidae Chamberlin, 1919

Genus Dorvillea Parfitt, 1866

Dorvillea rubra ${ }^{3}$ (Grube, 1856)

Dorvillea rubra: Grube, 1856; Perkins, 1998; Salazar-

Vallejo y Londoño-Mesa, 2004; Hernández-Alcántara et al., 2014.

Material examined: DOR0001, DOR0002, DOR0003, DOR0003, DOR0004, DOR0005, DOR0006.

Distribution: Caribbean, Florida, Gulf of Mexico, TEP.

Family Eunicidae Berthold, 1827

Genus Eunice Cuvier, 1817

Eunice cedroensis $^{3}$ Fauchald, 1970

Eunice cedroensis: Fauchald, 1970; Salazar-Vallejo y Londoño-Mesa, 2004.

Material examined: EUN0035, EUN0036, EUN0037, EUN0038.

Distribution: Gulf of Mexico, TEP.

Discussion: Although the specimens matched the description by Fauchald (1970), the setae illustrated in Figure E (Plate 2) were not observed.

Genus Lysidice Lamarck, 1818

Lysidice ninetta ${ }^{1}$ Audouin \& H Milne Edwards, 1833

Lysidice ninetta: Audouin y Milne, 1833; Monro, 1928; Fauchald, 1970, 1977; Laverde-Castillo, 1986; HernándezAlcántara y Solís-Weiss, 1991; de León-González et al., 1993; Gómez et al., 1997; Cantera et al., 1999; SalazarVallejo y Londoño-Mesa, 2004.

Material examined: EUN0040, EUN0041, EUN0042

Distribution: TEP, Colombia, Gulf of Mexico, Cuba, Belize, Belgium, Caribbean Sea, France, Greece, Jamaica, New Zealand, Red Sea, Spain, United Kingdom. 
Género Palola Gray in Stair, 1847

Palola paloloides $^{2}$ (Moore, 1909)

Palola paloloides: Moore, 1909; Fauchald, 1970, 1992;

Kudenov, 1975; Laverde-Castillo, 1986; Hernández-

Alcántara y Solís-Weiss, 1991; Salazar-Vallejo y LondoñoMesa, 2004.

Material examinado: EUN0043.

Distribución: San Diego, California; PTO, Pacífico colombiano.

Familia Flabelligeridae de Saint-Joseph, 1894

Género Semiodera Chamberlin, 1919

Semiodera cariboum ${ }^{3}$ (Grube, 1859)

Semiodera cariboum: Hartman, 1956; Salazar-Vallejo y

Londoño-Mesa, 2004.

Material examinado: FLA0006.

Distribución: Antillas, mar Caribe, golfo de México, PTO.

Discusión: Salazar-Vallejo y Londoño-Mesa (2004) la

registran para el PTO (S. cariboa) como una especie

dudosa para la región; sin embargo, cumple con las descripciones de Grube (1858) y de León-González et al. (2009).

Familia Nereididae Blainville, 1818

Género Eunereis Malmgre, 1865

Eunereis cf. longipes ${ }^{3}$ Hartman, 1936

Eunereis cf. longipes: Hartman, 1936; Salazar-Vallejo y

Londoño-Mesa, 2004.

Material examinado: NER0001, NER0002, NER0003, NER0004.

Distribución: California, PTO.

Discusión: Los especímenes observados coinciden con la descripción de Hartman (1936). Sin embargo, a pesar de presentar falcígeros homogonfos notopodiales de lámina terminada en punta aguda (Hartman 1936, Figura 53), la hoja del falcígero estudiado es más larga y el diente más agudo. Así mismo, en la Figura 53F (Hartman, 1936), se aprecian dientes a lo largo de toda la hoja de la seta mientras que en los especímenes colombianos tales dientes solo se observan en la región distal de la seta y el primer diente es más largo y rodea el diente del falcígero.
Genus Palola Gray in Stair, 1847

Palola paloloides ${ }^{2}$ (Moore, 1909)

Palola paloloides: Moore, 1909; Fauchald, 1970, 1992;

Kudenov, 1975; Laverde-Castillo, 1986; Hernández-

Alcántara y Solís-Weiss, 1991; Salazar-Vallejo y Londoño-

Mesa, 2004.

Material examined: EUN0043.

Distribution: San Diego, California; TEP, Colombian

Pacific.

Family Flabelligeridae de Saint-Joseph, 1894

Genus Semiodera Chamberlin, 1919

Semiodera cariboum ${ }^{3}$ (Grube, 1859)

Semiodera cariboum: Hartman, 1956; Salazar-Vallejo y Londoño-Mesa, 2004.

Material examined: FLA0006.

Distribution: Antilles, Caribbean Sea, Gulf of Mexico, TEP.

Discussion: Salazar-Vallejo and Londoño-Mesa (2004) register this as a dubious species for the TEP (S. cariboa); however, the observed specimen matched the descriptions by Grube (1858) and de León-González et al. (2009).

Family Nereididae Blainville, 1818

Genus Eunereis Malmgre, 1865

Eunereis cf. longipes ${ }^{3}$ Hartman, 1936

Eunereis cf. longipes: Hartman, 1936; Salazar-Vallejo y Londoño-Mesa, 2004.

Material examined: NER0001, NER0002, NER0003, NER0004.

Distribution: California, TEP.

Discussion: Observed specimens matched the description by Hartman (1936); however, although having notopodial laminar homogonfous falcigerous ending in an acute tip (Hartman, 1936, Figure 53), the falcigerous lamina is longer and the tooth more acute. Likewise, in Figure 53F (Hartman, 1936), teeth can be seen along the whole setae sheet, while in the Colombian specimens, the teeth were only observed in the distal portion of the setae and the first tooth is longer and surrounds the falcigerous tooth. 
Familia Phyllodocidae Örsted, 1843

Género Eteone Savigny, 1822

Eteone cf. pacifica ${ }^{3}$ Hartman, 1936

Eteone cf. pacifica: Reish, 1963; Salazar-Vallejo y

Londoño-Mesa, 2004.

Material examinado: PHY0008, PHY0009.

Distribución: Friday Harbor (isla San Juan), PTO.

Discusión: Los especímenes observados coinciden con la descripción de León-González et al. (2009); sin embargo, los cirros ventrales no presentan el mismo largo que los dorsales y tampoco presentaron una forma auricular definida. Lo anterior puede deberse a que, cuando estos organismos se fijan, los caracteres pueden sufrir leves modificaciones.

Familia Sabellidae Latreille, 1825

Género Euratella Chamberlin, 1919

Euratella salmacidis ${ }^{3}$ (Claparède, 1869)

Euratella salmacidis: Claparède, 1869; de León-González et al., 1993; Salazar-Vallejo y Londoño-Mesa, 2004.

Material examinado: SAL0005, SAL0006, SAL0007, SAL0008, SAL0009, SAL0010.

Distribución: mar Mediterráneo, océano Atlántico Norte, España, PTO.

Familia Spionidae Grube, 1850

Género Dipolydora Verril, 1881

Dipolydora commensalis ${ }^{3}$ (Andrews, 1891)

Dipolydora commensalis: Andrews, 1891; Hartman, 1941; Salazar-Vallejo y Londoño-Mesa, 2004.

Material examinado: SPI0011, SPI0012, SPI0013, SPI0014, SPI0015, SPI0016.

Distribución: Carolina del Norte, mar del Japón, océano Atlántico Norte, PTO.

Género Polydora Bosc, 1802

$$
\text { Polydora ciliata }{ }^{3} \text { (Johnston, 1838) }
$$

Polydora ciliata: Johnston, 1838; Hartman, 1941;

Mustaquim, 1986; Salazar-Vallejo y Londoño-Mesa, 2004.

Material examinado: SPI0019.

Distribución: Reino Unido, Francia, golfo de Nápoles, mar Negro, golfo de Adén, océano Atlántico Norte, Bélgica, bahía de Fundy, canal Inglés, Grecia, Irlanda, Madagascar,
Family Phyllodocidae Örsted, 1843

Genus Eteone Savigny, 1822

Eteone cf. pacifica $^{3}$ Hartman, 1936

Eteone cf. pacifica: Reish, 1963; Salazar-Vallejo y

Londoño-Mesa, 2004.

Material examined: PHY0008, PHY0009.

Distribution: Friday Harbor (San Juan Island), TEP.

Discussion: Observed specimens matched the description by de León-González et al. (2009); however, ventral and dorsal cirri differ in longitude and lack a well-defined auricular shape. This can be due to slight changes in soft tissues during fixation.

Family Sabellidae Latreille, 1825

Genus Euratella Chamberlin, 1919

Euratella salmacidis ${ }^{3}$ (Claparède, 1869)

Euratella salmacidis: Claparède, 1869; de León-González et al., 1993; Salazar-Vallejo y Londoño-Mesa, 2004.

Material examined: SAL0005, SAL0006, SAL0007, SAL0008, SAL0009, SAL0010.

Distribution: Mediterranean Sea, North Atlantic Ocean, Spain, TEP.

Family Spionidae Grube, 1850

Genus Dipolydora Verril, 1881

Dipolydora commensalis ${ }^{3}$ (Andrews, 1891)

Dipolydora commensalis: Andrews, 1891; Hartman, 1941; Salazar-Vallejo y Londoño-Mesa, 2004.

Material examined: SPI0011, SPI0012, SPI0013, SPI0014, SPI0015, SPI0016.

Distribution: North Carolina, Japan Sea, North Atlantic Ocean, TEP.

Genus Polydora Bosc, 1802

Polydora ciliata ${ }^{3}$ (Johnston, 1838)

Polydora ciliata: Johnston, 1838; Hartman, 1941;

Mustaquim, 1986; Salazar-Vallejo y Londoño-Mesa, 2004.

Material examined: SPI0019.

Distribution: United Kingdom, French, Gulf of Naples, Black Sea, Gulf of Aden, North Atlantic Ocean, Belgium, Bay of Fundy, English Channel, Greece, Ireland, 
Mozambique, Países Bajos, mar del Norte, mar Rojo, España, Ucrania, PTO.

Discusión: El espécimen observado coincide con las descripciones de Johnston (1838) y Mustaquim (1986), con la excepción de que no se observaron los cuatro ojos que reporta Mustaquim (1986).

Familia Syllidae Grube, 1850

Género Cicese Díaz-Castañeda \& San Martín, 2001

Cicese cf. sphaerosylliformis ${ }^{3}$ Díaz-Castañeda \&

San Martín, 2001

Cicese cf. sphaerosylliformis: Díaz-Castañeda y San Martín, 2001; Díaz-Castañeda et al., 2005; HernándezAlcántara et al., 2008.

Material examinado: SYL0011.

Distribución: costa Pacífica de Baja California (México).

Discusión: El espécimen observado coincide con la descripción de Díaz-Castañeda y San Martín (2001); sin embargo, en los falcígeros la distancia que se observa entre los dientes distales y los basales es más corta que la presentada en la ilustración. En la seta simple no se observan los dientes ilustrados en Díaz-Castañeda y San Martín (2001, Figura 5G, 5H, 5I).

Género Dentatisyllis Perkins, 1981

Dentatisyllis cf. carolinae ${ }^{3}$ (Day, 1973)

Dentatisyllis cf. carolinae: Day, 1973; Perkins, 1980; de León-González et al., 2009.

Material examinado: SYL0012, SYL0013.

Distribución: golfo de México, océano Atlántico Norte.

Discusión: de León-González et al. (2009) registran un trepan con aproximadamente 10 dientes mientras que en los especímenes estudiados se observaron 8. Para los cirros dorsales, de León-González et al. (2009) mencionan más de 30 artejos mientras que en los especímenes estudiados se observaron aproximadamente 20 artejos. Finalmente, Day (1973, figura 4E) y Perkins (1980, Figura 38A) ilustran setas simples que no se observaron en los especímenes estudiados.

Género Syllis Lamarck, 1818

Syllis gracilis $^{1}$ Grube, 1840

Syllis gracilis: Grube, 1840; Monro, 1933; Fauchald, 1977; Laverde-Castillo, 1986; Salazar-Vallejo et al., 1990; Estape y San Martín, 1991; Hernández-Alcántara y Solís-Weiss,
Madagascar, Mozambique, Netherlands, North Sea, Red Sea, Spain, Ukraine, TEP.

Discussion: The observed specimen matched the descriptions by Johnston (1838) and Mustaquim (1986), with the only exception that the four eyes reported by Mustaquim (1986) were not observed in our specimen.

Family Syllidae Grube, 1850

Genus Cicese Díaz-Castañeda \& San Martín, 2001

Cicese cf. sphaerosylliformis ${ }^{3}$ Díaz-Castañeda \& San Martín, 2001

Cicese cf. sphaerosylliformis: Díaz-Castañeda y San Martín, 2001; Díaz-Castañeda et al., 2005; HernándezAlcántara et al., 2008.

Material examined: SYL0011.

Distribution: Pacific Coast of Baja California (Mexico).

Discussion: The observed specimen matched the description in Díaz-Castañeda and San Martín (2001), but the distance between distal and basal falcigerous teeth is shorter than the one presented in their illustration. Besides, in the simple setae, there are no teeth present as shown in the illustration by Díaz-Castañeda and San Martín (2001, Figure 5G, 5H, 5I).

Genus Dentatisyllis Perkins, 1981

Dentatisyllis cf. carolinae ${ }^{3}$ (Day, 1973)

Dentatisyllis cf. carolinae: Day, 1973; Perkins, 1980; de León-González et al., 2009.

Material examined: SYL0012, SYL0013.

Distribution: Gulf of Mexico, North Atlantic Ocean.

Discussion: de León-González et al. (2009) register a trepan with approximately 10 teeth, while in our specimens, only 8 teeth were observed. For the articulated dorsal cirri, they mention 30 segments, while in our specimens we observed only approximately 20 segments. Finally, the simple setae illustrated by Day (1973, Figure 4E) and Perkins (1980, Figure 38A) were not observed in our specimens.

Genus Syllis Lamarck, 1818

Syllis gracilis ${ }^{1}$ Grube, 1840

Syllis gracilis: Grube, 1840; Monro, 1933; Fauchald, 1977; Laverde-Castillo, 1986; Salazar-Vallejo et al., 1990; Estape y San Martín, 1991; Hernández-Alcántara y Solís-Weiss, 
1991; de León-González et al., 1993; Bastida-Zavala, 1995; Capa et al., 2001; Hernández-Alcántara et al., 2003; Salazar-Vallejo y Londoño-Mesa, 2004.

Material examinado: SYL0018, SYL0019, SYL0020, SYL0021, SYL0022.

Distribución: golfo de Nápoles, mar Mediterráneo, mar Negro, región chilena del océano Pacífico Sur, región yibutiana del golfo de Adén, región yibutiana del mar Rojo, Antillas, costa Occidental de África, golfo de México, mar Caribe, Trinidad y Tobago, España, Reino Unido, Italia, Irlanda, Grecia, PTO.

Género Syllis Lamarck, 1818

Syllis prolifera ${ }^{3}$ Krohn, 1852

Syllis prolifera: López et al., 1997; Capa et al., 2001; Salazar-Vallejo y Londoño-Mesa, 2004.

Material examinado: SYL0017.

Distribución: Antillas, mar Negro, región yibutiana del golfo de Aden, región yibutiana del mar Rojo, mar Caribe, Cuba, golfo de México, Italia, Madagascar, Nueva Zelanda, océano Atlántico Norte, España, Trinidad y Tobago, Reino Unido, Grecia, PTO.

\section{Sipuncula}

\section{Clase Phascolosomatidea}

Familia Aspidosiphonidae Quatrefages, 1865

Género Aspidosiphon Diesing, 1851

Aspidosiphon (Aspidosiphon) cf. elegans ${ }^{3}$

(Chamisso \& Eysenhardt, 1821)

Aspidosiphon (Aspidosiphon) cf. elegans: Chamisso y Eysenhardt, 1821; Cutler, 1994.

Material examinado: ASP0001, ASP0002, ASP0003, ASP0004, ASP0005, ASP0006, ASP0007.

Distribución: mar Mediterráneo-Cuenca Oriental, mar Egeo, golfo de México, Madagascar, océano Atlántico Norte, mar Rojo, océano Pacífico Sur.

Familia Phascolosomatidae Stephen \& Edmonds, 1972

Género Phascolosoma Leuckart, 1828

Phascolosoma (Phascolosoma) cf. nigrescens ${ }^{3}$

(Keferstein, 1865)

Phascolosoma (Phascolosoma) cf. nigrescens: Fisher, 1952; Cutler, 1994.
1991; de León-González et al., 1993; Bastida-Zavala, 1995; Capa et al., 2001; Hernández-Alcántara et al., 2003; Salazar-Vallejo y Londoño-Mesa, 2004.

Material examined: SYL0018, SYL0019, SYL0020, SYL0021, SYL0022.

Distribution: Gulf of Naples, Mediterranean Sea, Black Sea, Chilean part of the South Pacific Ocean, Djiboutian region of the Gulf of Aden, Djiboutian region of the Red Sea, Antilles, West Africa Coast, Gulf of Mexico, Caribbean Sea, Trinidad and Tobago, Spain, United Kingdom, Italy, Ireland, Greece, TEP.

Genus Syllis Lamarck, 1818

Syllis prolifera $^{3}$ Krohn, 1852

Syllis prolifera: López et al., 1997; Capa et al., 2001; Salazar-Vallejo y Londoño-Mesa, 2004.

Material examined: SYL0017.

Distribution: Antilles, Black Sea, Djiboutian part of the Gulf of Aden, Djiboutian part of the Red Sea, Caribbean Sea, Cuba, Gulf of Mexico, Italy, Madagascar, New Zealand, North Atlantic Ocean, Spain, Trinidad and Tobago, United Kingdom, Greece, TEP.

\section{Sipuncula}

\section{Class Phascolosomatidea}

Family Aspidosiphonidae Quatrefages, 1865

Genus Aspidosiphon Diesing, 1851

Aspidosiphon (Aspidosiphon) cf. elegans ${ }^{3}$

(Chamisso \& Eysenhardt, 1821)

Aspidosiphon (Aspidosiphon) cf. elegans: Chamisso y Eysenhardt, 1821; Cutler, 1994.

Material examined: ASP0001, ASP0002, ASP0003, ASP0004, ASP0005, ASP0006, ASP0007.

Distribution: Mediterranean Sea-Eastern Basin, Aegean Sea, Gulf of Mexico, Madagascar, North Atlantic Ocean, Red Sea, South Pacific Ocean.

Family Phascolosomatidae Stephen \& Edmonds, 1972 Genus Phascolosoma Leuckart, 1828

Phascolosoma (Phascolosoma) cf. nigrescens ${ }^{3}$ (Keferstein, 1865)

Phascolosoma (Phascolosoma) cf. nigrescens: Fisher, 1952; Cutler, 1994. 
Material examinado: PHS0001, PHS0002, PHS0003.

Distribución: Madagascar, mar Rojo, golfo de México.

Phascolosoma (Phascolosoma) cf. perlucens

Baird, 1868

Phascolosoma (Phascolosoma) cf. perlucens: Cutler, 1994; Cantera et al., 2003; Kawauchi y Giribet, 2010.

Material examinado: PSH0004, PSH0005, PSH0006, PSH0007, PSH0008, PSH0009, PSH0010.

Distribución: Madagascar, golfo de México, isla Gorgona (Colombia).

Discusión: Los especímenes observados cumplen con la descripción de Kawauchi y Giribet (2010); sin embargo, se utiliza cf. debido a que, de acuerdo con los autores mencionados, existe necesidad de aclarar la taxonomía de esta especie con análisis moleculares, pues los caracteres morfológicos dentro del género son muy variables y no son completamente útiles para la identificación correcta de las especies.

\section{AGRADECIMIENTOS}

Los autores quieren agradecer a los funcionarios del Parque Nacional Natural Gorgona por su continuo apoyo logístico, especialmente a María Ximena Zorrilla (jefe del Parque), Luís Payán (biólogo del Parque) y Héctor Chirimía González (guardaparques). También quieren agradecer a Mario Hernán Londoño por instruir a M.F. Cardona-Gutiérrez en la taxonomía de poliquetos y a Itzahi Silva Morales por su colaboración en la identificación de los sipúnculos. Finalmente, los autores quieren agradecer al Grupo de Investigación en Ecosistemas Rocosos Intermareales y Submareales Someros (LITHOS) de la Universidad del Valle por su apoyo en campo. Esta investigación es parte del proyecto cofinanciado por Colciencias (1106-659-44216) y la Universidad del Valle (CI-71002). Contribución 17 del Instituto de Ciencias del Mar y Limnología (Incimar) de la Universidad del Valle.
Material examined: PHS0001, PHS0002, PHS0003.

Distribution: Madagascar, Red Sea, Gulf of Mexico. Phascolosoma (Phascolosoma) cf. perlucens Baird, 1868

Phascolosoma (Phascolosoma) cf. perlucens: Cutler, 1994; Cantera et al., 2003; Kawauchi y Giribet, 2010.

Material examined: PSH0004, PSH0005, PSH0006, PSH0007, PSH0008, PSH0009, PSH0010.

Distribution: Madagascar, Gulf of Mexico, Gorgona Island (Colombia).

Discussion: The observed specimens matched the description by Kawauchi and Giribet (2010); however, we use cf. because, according to the aforementioned authors, there is a need for molecular analyses to discern this specie within the genus since morphological characters are highly variable and not fully useful for the correct identification of species.

\section{ACKNOWLEDGEMENTS}

The authors want to thank the Gorgona National Natural Park personnel for their continuous and valuable logistic support, especially Maria Ximena Zorrilla (Head of the Park), Luis Payán (Biologist of the Park), and Héctor Chirimía González (Park Ranger). We also want to thank Dr. Mario Hernán Londoño for instructing M.F. CardonaGutiérrez in polychaetes taxonomy and Itzahi Silva Morales for her support in sipunculid identification. We also want to thank the people of the Research Group on Intertidal and Shallow Subtidal Rocky Ecosystems (LITHOS) of Universidad del Valle for field support. This research is part of a larger project co-funded by Colciencias (1106-65944216) and Universidad del Valle (CI-71002). Contribution 17 from the Universidad del Valle's Institute of Marine Sciences and Limnology (Incimar). 


\section{BIBLIOGRAFÍA/LITERATURE CITED}

Abd-Elnaby, F.A. 2009. New records of Polychaetes from the south part of Suez Canal, Egypt. World J. Fish Mar. Sci., 1(1): 7-19.

Andrews, E.A. 1891. A commensal annelid. Am. Nat., 25(289): 25-35.

Audouin, J.V. and E.H. Milne. 1833. Classification des annélides et description de celles qui habitant les cotes de la France. Ann. Sci. Nat., 1(28): 187-247.

Bastida-Zavala, J.R. 1995. Poliquetos (Annelida: Polychaeta) del arrecife coralino de Cabo Pulmo-Los Frailes, B.C.S., México. Rev. Zool., 6: 9-29.

Berkeley, E. and C. Berkeley. 1954. Notes on the life-history of the polychaete Dodecaceria fewkesii (nom. n.). J. Fish. Res. Board Can., 11(3): 326-334.

Cantera, J., B. Thomassin, and P.M. Arnaud. 1999. Faunal zonation and assemblages in the Pacific Colombian mangroves. Hydrobiologia, 413: 17-33. https://doi.org/10.1023/A:1003890826741

Cantera, J., C. Orozco, E. Londoño-Cruz, and G. Toro-Farmer. 2003. Coral reef paper abundance and distribution patterns of infaunal associates and macroborers of the branched coral (Pocillopora damicornis) in Gorgona Island (Eastern Tropical Pacific). Bull. Mar. Sci., 72(1): $207-219$.

Capa, M., G. San Martín y E. López. 2001. Syllinae (Syllidae: Polychaeta) del Parque Nacional de Coiba, Panamá. Rev. Biol. Trop., 49: 103-115.

Chamberlin, R.V. 1919. The Annelida Polychaeta of the Albatross Tropical Pacific Expedition, 1891-1905. Mem. Mus. Comp. Zool. Harvard Coll., 48: $1-514$.

Chamisso, A. and C.G. Eysenhardt. 1821. De animalibus quibusdam e classe vermium Linneana, in circumnavigatione Terrae, auspicante Comite N. Romanoff, duce Ottone di Kotzebue, annis 1815-1818 peracte, observatis Fasciculus scundus, reliquos vermes continens. Nova Acta Physico-med. Acad. Cesareae Leopoldino-Carolinae Nat. Curios., 10: 343-373.

Choi, H.K., H. Kim, and S.M. Yoon. 2018. Timarete posteria, a new cirratulid species from Korea (Annelida, Polychaeta, Cirratulidae). Zookeys, 806: 1.

Claparède, É. 1869. Les Annélides Chétopodes du Golfe de Naples. Seconde partie. Ordre II. Annélides Sédentaires (Aud. et Edw.). Mém. Soc. Phys. Genève, 20(1): 1-225.

Cutler, E.B. 1994. The Sipuncula: their systematics, biology and evolution. Cornell University, New York. 453 p.

Day, J.H. 1973. New Polychaeta from Beaufort: with a key to all species recorded from North Carolina. U.S. Dept. Com., Nat. Ocean. Atmos. Adm., Nat. Mar. Fish. Serv., Seattle. 140 p.

de León-González, J.A., A. Leija-Tristán y S.I. Salazar-Vallejo. 1993. Epifauna del ostión espinoso Spondylus princeps unicolor (Mollusca: Bivalvia), de Puerto Escondido, Golfo de California, México. Rev. Biol. Trop., 41: 877-881.

de León-González, J.A., J.R. Bastida-Zavala, L.F. Carrera-Parra, M.E. García-Garza, A. Peña-Rivera, S. I. Salazar-Vallejo y V. Solís-Weiss. 2009. Poliquetos (Annelida: Polychaeta) de México y América Tropical. Universidad Autónoma de Nuevo León, Monterrey. 737 p.

Díaz, J.M., L.M. Barrios, M.H. Cendales, J. Garzón-Ferreira, J. Geister, F. Parra-Velandia y F.A. Zapata. 2000. Áreas coralinas de Colombia. Ser. Publ. Espec., (5). Invemar, Santa Marta. 176 p.

Díaz-Castañeda, V. and G. San Martín. 2001. Syllidae (Polychaeta) from San Quintín lagoon, Baja California, Mexico, with the description of a new genus. Proc. Biol. Soc. Wash., 114(3): 708-719.

Díaz-Castañeda, V., J.A. de León-González, and E.S. Arellano. 2005. Structure and composition of the polychaete community from bahía San Quintín, Pacific coast of Baja California, Mexico. Bull. South. Calif. Acad. Sci., 104(2): 75-99.

Estape, S. y G. San Martín. 1991. Descripción de los estolones reproductores de algunas especies de la subfamilia Syllinae (Polychaeta, Syllidae). Misc. Zool., 15: 43-62.

Fauchald, K. 1970. Polychaetous annelids of the families Eunicidae, Lumbrinerida, Iphitimidae, Arabellidae, Lysaretidae and Dorvilleidae from Western Mexico. Allan Hancock Monogr. Mar. Biol., 5: 1-335.

Fauchald, K. 1977. Polychaetes from intertidal areas in Panama, with a review of previous shallow-water records. Smithson. Contrib. Zool., 221 : 1-81.

Fauchald, K. 1992. A review of the genus Eunice (Polychaeta: Eunicidae) based upon type material. Smithson. Contrib. Zool., 523: 1-422.

Fisher, W.K. 1952. The sipunculid worms of California and Baja California. Proc. US Nat. Mus., 102(3306): 371-450. https://doi.org/10.5479/ si.00963801.102-3306.371

Gibson, P.H. 1978. Systematics of Dodecaceria (Annelida: Polychaeta) and its relation to the reproduction of its species. Zool. J. Linn. Soc., 63(3): $275-287$.

Giraldo, A. 2012. Geomorfología e hidroclimatología de isla Gorgona. 17-23. In: Giraldo, A. y B. Valencia (Eds.). Isla Gorgona: paraíso de biodiversidad y ciencia. Univ. Valle, Cali. 226 p. 
Giraldo, A., E. Rodríguez-Rubio y F.A. Zapata. 2008. Condiciones oceanográficas en isla Gorgona, Pacífico oriental tropical de Colombia. Lat. Am. J. Aquat. Res., 36(1): 121-128.

Gómez, P., J.A. Mercado, L.M. Mitchell y S.I. Salazar-Vallejo. 1997. Poliquetos de fondos duros (Polychaeta) de bahías de Huatulco y Puerto Ángel, Oaxaca, México. Rev. Biol. Trop., 45: 1067-1074.

Grube, A.E. 1840. Actinien, Echinodermen und Würmer des Adriatischen-und Mittelmeers nach eigenen Sammlungen beschrieben. J.H. Bon, Königsberg. 93 p. https://doi.org/10.5962/bhl.title.10133

Grube, A.E. 1857. Annulata Örstediana. Enumeratio Annulatorum, quae in itinere por Indiam occidentalem er Americam centralem annis 1845-1848 suscepto legit cl. A.S. Örsted, adjectis speciebus nonnullis a cl. H. Kröyero in itinere ad Americam meridionalem callectis. Part 1. Videnskabelige Meddelelser fra Dansk naturhistorik Forening I Köbenhavn, 1856: 44-62.

Grube, A.E. 1859. Annulata Örstediana. Enumeratio Annulatorum, quae in itinere Indiam Occidentalem et Ammericam Centralem annis $1845-1848$ suscepto legit cl. A.S. Örsted, adjectis speciebus nonnullis a cl. H. Kroyero in itinere ad Americam meridionalem collectis. Part. 3. 105-120. Videnskabelige Meddelelser fra Dansk naturhistorik Forening I Köbenhavn, 1858: 105-120.

Hartman, O. 1936. New species of polychaetous annelids of the family Nereidae from California. Proc. US Nat. Mus., 83(2994): 467-480.

Hartman, O. 1941. Some contributions to the biology and life history of Spionidae from California. Allan Hancock Pac. Exp., 7: 289-323.

Hartman, O. 1954. Marine annelids from the northern Marshall Islands. Bikini and nearby Atolls, Marshall Islands. Geol. Surv. Profess. Paper, 260-Q: 619644.

Hartman, O. 1956. Polychaetous annelids erected by Treadwell, 1981 to 1984, together with a brief chronology. Bull. Am. Mus. Nat. Hist., 109: 239-310.

Hernández-Alcántara, P.L. and V. Solís-Weiss. 1991. New records of errant polychaetous annelids from the Gulf of California. Bull. Mar. Sci., 48: 251-260.

Hernández-Alcántara, P., J.D. Cortés-Solano, N.M. Medina-Cantú, A.L. Avilés-Díaz, and V. Solís-Weiss. 2014. Polychaete diversity in the estuarine habitats of Términos Lagoon, southern Gulf of Mexico. Mem. Mus. Vic., 71: 97-107.

Hernández-Alcántara, P., M.A. Tovar-Hernández, and V. Solís-Weiss. 2008. Polychaetes (Annelida: Polychaeta) described for the Mexican Pacific: an historical review and an update checklist. Lat. Am. J. Aquat. Res., 36(1): 37-61. https://doi.org/10.3856/vol36-issue1-fulltext-4

Hernández-Alcántara, P.L., S.C. Frontana-Uribe, and V. Solís-Weiss. 2003. Commented checklist of the polychaetes (Annelida: Polychaeta) from areas adjacent to islands of the Mexican Pacific. Bull. South. Calif. Acad. Sci., 102(1): 1-16.

Hutchings, P.A. 1986. Biological destruction of coral reefs. Coral Reefs, 4: 239-252.

Johnston, G. 1838. Miscellanea Zoologica. III. The British Ariciadae. Mag. Zool. Bot., Edinburgh, 2: 63-73, plate III. https://biodiversitylibrary.org/ page $/ 40025966$

Kawauchi, G.Y. and G. Giribet. 2010. Are there true cosmopolitan sipunculan worms? A genetic variation study within Phascolosoma perlucens (Sipuncula, Phascolosomatidae). Mar. Biol., 157(7): 1417-1431.

Kudenov, J.D. 1975. Errant polychaetes from the Gulf of California, Mexico. J. Nat. Hist., 9(1): 65-91.

Laverde-Castillo, J.J. 1986. Lista anotada de los poliquetos (Annelida) registrados para el Pacífico colombiano, con notas preliminares sobre su zoogeografía. Actual. Biol., 15(58): 123-130.

Londoño-Cruz, E., J. R. Cantera, G. Toro-Farmer, and C. Orozco. 2003. Internal bioerosion by macroborers in Pocillopora spp. in the tropical eastern Pacific. Mar. Ecol. Prog. Ser., 265: 289-295.

López, E., G. San Martín, P. Cladera de Lacodina y M. Capa. 1997. La fauna de anélidos poliquetos del Parque Nacional de Coiba (Panamá). 57-73. In: Castroviejo, S. y M. Velayos. (Ed.). Flora y fauna del Parque Natural de Coiba (Panamá), Inventario preliminar. Agencia Española de Cooperación Internacional, Madrid. $534 \mathrm{p}$.

Magalhaes, W.F. and J.H. Bailey-Brock. 2013. Bitentaculate Cirratulidae (Annelida: Polychaeta) from the northwestern Pacific Islands with description of nine new species. Zootaxa, 3630(1): 80-116.

Monro, C.C.A. 1928. Polychaeta of the families Polynoidae and Acoetidae from the vicinity of the Panama Canal, Collected by Dr. C. Crossland and Dr. Th. Mortensen. Zool. J. Linn. Soc., 36: 553-576.

Monro, C.C.A. 1933. The Polychaeta Sedentaria collected by Dr C. Crossland at Colón, in the Panama Region, and the Galapagos Islands during the expedition of the S.Y. St. George. Proc. Zool. Soc. Lond., 103(4): 1039-1092. https://doi.org/10.1111/j.1096-3642.1933.tb01640.x

Moore, J.P. 1904. New Polychaeta from California. Proc. Acad. Nat. Sci. Phila., 56: 484-503.

Moore, J.P. 1909. Polychaetous annelids from Monterey Bay and San Diego, California. Proc. Acad. Nat. Sci. Phila., 61: 235-295. 
Mustaquim, J. 1986. Morphological variation in Polydora ciliate complex (Polychaeta: Annelida). Zool. J. Linn. Soc., 86(1): 75-88.

Perkins, T.H. 1980. Syllidae (Polychaeta), principally from Florida, with descriptions of a new genus and twenty-one new species. Proc. Biol. Soc. Wash., 93(4): 1080-1172.

Perkins, T.H. 1998. Checklist of shallow-water marine polychaetous Annelida of Florida. In Camp, D., W. Lyons, and T. Perkins. (Eds). Checklist of selected shallow-water marine invertebrates of Florida. Florida Mar. Res. Inst. Tech. Rep., 3: 79-122.

Prahl, H. von y H. Erhardt. 1985. Colombia: corales y arrecifes coralinos. FEN Colombia, Bogotá. 295 p.

Prahl, H. von, F. Guhl y M. Grögl. 1979. Poliquetos de Gorgona. 131-140. In Prahl, H. von, F. Guhl, and M. Grögl. (Eds). Gorgona. Futura, Bogotá, D.C.

Reish, D.J. 1963. A quantitative study of the benthic polychaetous annelids of Bahia de San Quintín, Baja California. Pac. Nat., 3: 401-436.

Salazar-Vallejo, S.I. y M.H. Londoño-Mesa. 2004. Lista de especies y bibliografía de poliquetos (Polychaeta) del Pacífico Oriental Tropical. An. Inst. Biol. ser. Zool., 75(1): 9-97.

Salazar-Vallejo, S.I., J.A. de León-González y J.C. Chávez-Comparan. 1990. Poliquetos (Annelida: Polychaeta) de la Bahía de Manzanillo, con una clave ilustrada para las especies de Colima, México. Rev. Biol. Trop., 38(2A): 211-229.

White, J.K. 1980. Distribution, recruitment and development of the borer community in dead coral on shallow Hawaiian reefs. University of Hawaii, Honolulu. 238 p. http://hdl.handle.net/10125/18150

WoRMS. 2019. World Register of Marine Species. http://www.marinespecies.org 09/07/2019.

Zapata, F.A. 2001. Formaciones coralinas de isla Gorgona: 27-40. In: Barrios, L.M. y M. López-Victoria. (Eds.). Gorgona marina: contribución al conocimiento de una isla única. Ser. Publ. Esp., (7). Invemar, Santa Marta. 160 p.

Zapata, F.A. and Y.A. Morales. 1997. Spatial and temporal patterns of fish diversity in a coral reef at Gorgona Island, Colombia. Proc. Int. Coral Reef Symp., 1: $1029-1034$.

Zapata, F.A. and B. Vargas-Ángel. 2003. Corals and coral reefs of the Pacific coast of Colombia. 419-447. In: Cortés, J. (Ed.). Latin American Coral Reefs. Elsevier, Amsterdam. 508 p. https://doi.org/10.1016/B978-0-444-51388-5.X5000-0 